\title{
An effective physical developer (PD) method for use in Australian laboratories
}

Authors: Mackenzie de la Hunty ${ }^{1 *}$, Sébastien Moret $^{1}$, Scott Chadwick ${ }^{1}$, Chris Lennard ${ }^{2}$, Xanthe Spindler ${ }^{1}$, Claude Roux ${ }^{1}$

${ }^{1}$ Centre for Forensic Science, University of Technology Sydney, PO Box 123, Broadway, NSW 2007, Australia,

${ }^{2}$ School of Science and Health, Western Sydney University, Richmond, NSW 2753, Australia.

*Corresponding Author's email; Mackenzie.delahunty@uts.edu.au

\section{Abstract}

Physical developer (PD) is an underutilised technique for the development of latent marks on porous surfaces that have been wet, or as a subsequent technique in a development sequence. It is a multistep technique that works by selectively reducing silver ions to silver metal at nucleating sites in fingermark residue. Its use is associated with a plethora of issues, largely surrounding the inherent instability of the working solution. Recently, one of the components of the working solution,

Synperonic $\mathrm{N}$, has ceased in production, and the recommended replacement is Tween 20 . This paper addresses factors during PD processing using Tween 20 , other than reagent formulation that should be considered when using the technique.

\section{Introduction}

Physical developer (PD) is a latent fingermark development technique used for porous surfaces. It is used after treatment with amino acid sensitive techniques, as well as on porous substrates that have been wet where amino acid sensitive techniques would be ineffective (due to the removal of watersoluble amino acids). Issues during fingermark development have acted as a deterrent to the routine use of the technique for volume crime exhibits and, as such, it is usually reserved for major crime cases [1]. The PD technique has been shown to develop marks that remained undetected using other techniques [2,3], and results in grey/silver ridges in the pattern of the latent fingermark. Development can be of only the ridges, the ridges and the pores, or solely just at pore sites (Figure 1) [4].
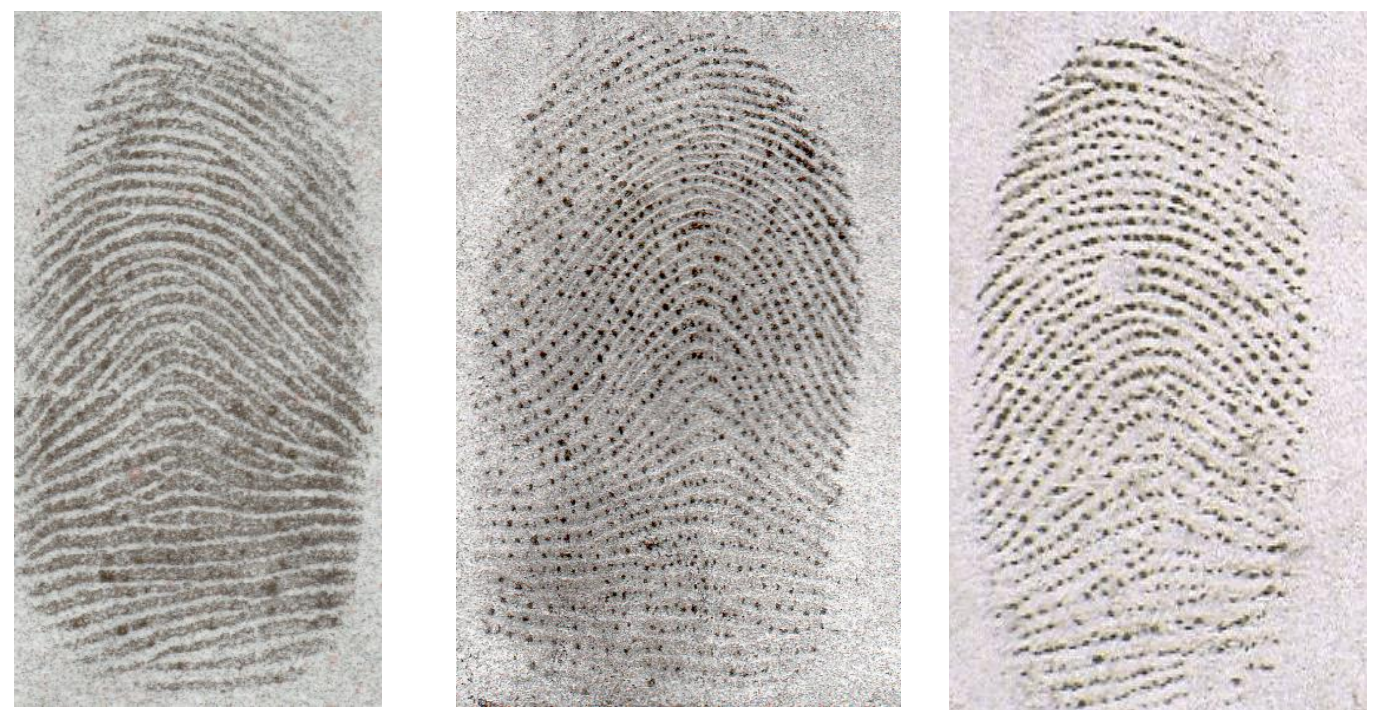

Figure 1: Physical developer shows varied types of development on the ridges (left), ridges and pore sites (centre) and pore sites (right) 
Early formulations of the PD solution were adapted to include surfactants as a means to extend the shelf life of the solution, and to make it less reactive to contaminants. As a purposeful consequence, the solution was made to be more stable. The recent recommendations for the substitution of Synperonic $\mathrm{N}$ for Tween 20 in the dual surfactant-detergent solution that also contains $\mathrm{n}$ dodecylamine acetate (n-DDAA) [5], has resulted in a PD working solution that is very stable. The replacement of Synperonic $\mathbf{N}$ with Tween 20 is no longer a choice but an inevitability (due to termination of manufacture and safety concerns). Usually the stability of development solutions is favourable; however, the mechanism of PD relies on localised instability of the solution in contact with fingermark residue, to promote the destabilisation of silver ions and encourage the formation of silver metal [6].

Preliminary observations by our group have shown that the Tween 20 formulation results in the deposition of silver at a slower rate than the previous Synperonic $\mathrm{N}$ formulation [7], but gives comparable results when specific steps are taken during working solution preparation and processing. The increase in treatment time seems to have a slight effect on the contrast of the sample as, over time, more silver is deposited onto the background of the substrate, although this is not significantly detrimental to the development [4]. The surfactant concentration in the PD working solution has a direct effect on the speed and quality of development for treated fingermarks. In our research, it has been found that a 50\% reduction in surfactant concentration (when using Tween 20 as a replacement for Synperonic $\mathrm{N}$ ) in the detergent-surfactant solution leads to a decrease in development times without sacrificing development quality, as well as extending the shelf life of the working solution (compared to the Synperonic $N$ formulation) to well over three months $[4,8,9]$.

Discussions in the fingermark development community have indicated that some laboratories store PD working solutions containing all of the components, whilst some add in the detergent-surfactant and silver nitrate solutions immediately prior to use [10]. This paper discusses which of these storage conditions produces a more effective PD working solution on natural fingermarks. In addition to the inclusion of Tween 20 in the working solution, there are a number of general considerations that have been identified to facilitate the correct preparation of the working solution and its application to samples. The treatment method presented in this article has been successfully utilised by both staff and undergraduate students at the University of Technology Sydney (UTS).

\section{Chemical considerations}

The chemicals used in this research and available to our laboratory are listed here. Citric acid, maleic acid, and silver nitrate were obtained from BDH-Prolabo Chemicals (VWR International Pty Ltd, Australia). Ferric nitrate nonahydrate (Chem-Supply Pty Ltd, Australia), ammonium ferrous sulphate (Chem-Supply Pty Ltd, Australia), n-dodecylamine acetate (Optimum Technologies, Australia) and Tween 20 surfactant (Sigma-Aldrich, USA) were used as supplied. High-purity water was obtained from a high-throughput three-stage water filtration system (AKF 300 activated carbon filter; Bewades 58 LC UV disinfection system; Vertex SS-360HR reverse osmosis water purification system) that supplies all "deionised water" taps at the university.

\section{Equipment considerations}

If PD is used regularly, glass processing basins should be kept solely for PD processing to avoid potential contamination leading to working solution destabilisation. It is important to use processing basins (glass or non-porous stoneware) that are clean and free of scratches. If processing basins are not reserved solely for PD use, consider using plastic bags (check reactivity with PD working solution) to line the processing basins. It is important to wash the processing basins with a detergent in 
between uses with other development reagents, ensuring that the detergent is completely removed prior to use with PD. Rinse the glassware completely with deionised (DI) water 2-3 times, ensuring no trace of tap water remains. If processing basins are reserved solely for PD use, rinsing with DI water 2-3 times in between use is also necessary. Use a gloved hand to rub the corners of the tray to remove any deposited silver and do not use an abrasive sponge. After washing, let the glassware dry naturally, and do not dry with paper towel as it may leave residual fibres in the glassware that may affect the working solution.

When processing samples, do not use metal tweezers or tongs as they may damage the sample and cause silver deposition in the areas that have been touched. We have found the use of gloved hands (cotton gloves underneath clean nitrile gloves) to gently move the samples between the solutions to be the most effective handling method. Throughout the development steps, you should change your gloves regularly. Plastic tweezers may be utilised if preferred; however, localised compression of cellulose fibres may promote silver deposition in these areas, which can be avoided by using gloved hands.

\section{Processing considerations}

Five processing trays are recommended for treating exhibits using the PD method (Figure 2). If an exhibit is clean and has not undergone other development treatments, they should be left in tray 1 for 5-15 minutes, or until the article appears uniformly saturated; this allows for the maleic acid in the subsequent step to uniformly soak into the substrate. If an exhibit is dirty, dusty or has been through a sequence of developments, two washes of 10-15 min is required in tray 1 . Exhibits should be placed in tray 2 for 15-30 minutes, or until the substrate is uniformly opaque to ensure the removal of all carbonates from the paper. A more concentrated maleic acid solution will enable this to occur more quickly if desired. The remaining treatments are: tray 3 for 5-10 minutes with regular agitation to remove the maleic acid from the substrate; tray 4 for 5-40 minutes, or until sufficient development has been achieved; and tray 5 for 10 minutes twice with consistent agitation to ensure that the PD working solution has been removed from the exhibit. The major deviations to the published methods are different recommended treatment times and the addition of tray 3 , which allows for longer use of the PD working solution in tray 4, as maleic acid is not transferred from tray 2 if the article is washed with water in tray 3.

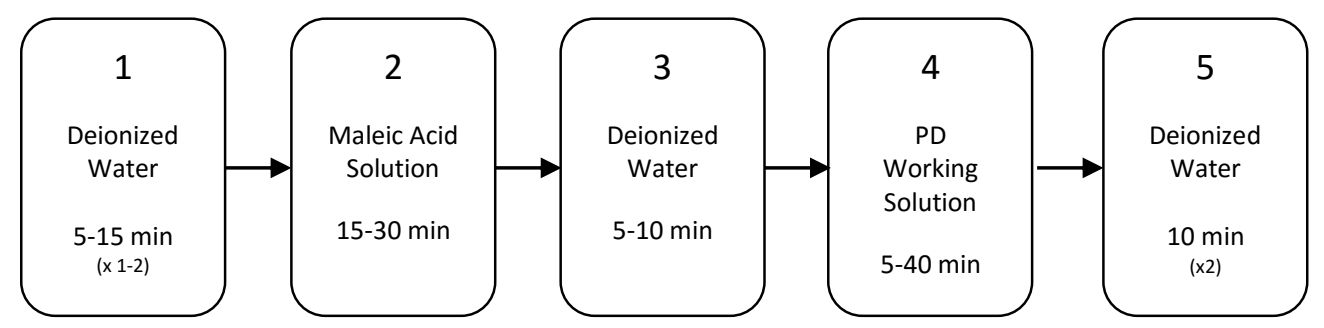

Figure 2: Processing basins for treatment of exhibits using physical developer in an Australian laboratory

Some protocols encourage the use of mechanical rockers or shakers during article immersion in the working solution [11]; however, gentle rocking of the trays by lifting one edge 1-2 cm slowly and repetitively is sufficient to ensure that the solution is "washing" the sample and penetrating the substrate. It is important to not consistently touch the sample with gloved hands to further 
submerge the document in the working solution, especially when samples are in the maleic acid wash basin, as this will result in sporadic development at sites of cellulose compression/disturbance.

\section{General considerations}

When using Tween 20 as a substitute for Synperonic N, working solutions should be aged for a minimum of 5 days to allow equilibration. The working solution should also be aged containing all components of the working solution, including the silver nitrate solution. Some institutions prefer to add in the silver nitrate immediately before use; however, this does not give the working solution sufficient time to equilibrate and sufficiently destabilise. Fresh PD solutions (less than 5 days old) tend to be more stable than aged PD solutions and this leads to longer development times [4].

Fresh PD solutions are more effective at developing fresh fingermarks (where the composition is relatively unstable and changing rapidly); aged PD solutions are more unstable and are more effective at developing aged fingermark residue (where the composition is more stable and unchanging). Although the age of the fingermark residue is unknown in casework samples, an understanding of this concept is important, and it explains why control samples of varying ages are necessary. If you are going to use PD regularly, create a "bank" of control fingermark samples on a paper substrate that does not adversely react with PD. Before you use the working solution on your casework samples, place a "fresh" control (0-24 hours old) and an "aged" control (at least 2 weeks old) into the solution to observe the effectiveness of the solution on both fresh and aged fingermarks.

Effective detergent-surfactant solutions are often described in procedural manuals as being clear and colourless; however, all of the solutions used in this research were cloudy. The cloudiness occurs during the solubilisation of the n-DDAA in water, and persists during storage (either in or out of the fridge), and during incorporation into a PD working solution. The cloudiness exhibited in the solution during this research is not milky, and appears to more closely resemble an almost homogenous, very fine suspension of solid white particles. This cloudiness may be due to incomplete solubilisation of the $n$-DDAA but it does not impact on the effectiveness of the solution. It is usually stated that if the detergent-surfactant solution appears cloudy then it is no longer effective. This may be due to impure n-DDAA being dispatched from chemical providers to laboratories in the US [12] and the UK [13]. The impure $n$-DDAA supplied to these laboratories resulted in a milky surfactant that did not produce a useable PD working solution. We did not encounter such issues.

\section{Conclusion}

Physical developer is an underutilised technique for latent fingermark development due to its perceived complexity and inconsistent results. Although procedural manuals tend to focus on formulations, this article discusses other considerations that should be taken into account when making up and using a PD working solution. There are a number of considerations that can aid in the utilisation of this technique for the successful development of latent fingermarks on porous surfaces, either at the end of a detection sequence or when the substrate has been wet. PD can develop fingermarks that remain undetected by other methods.

\section{References}

1. Byard, G., Personal Communication. 2014 
2. Raymond, J., Personal Communication. 2017

3. Braasch, K., de la Hunty, M., Deppe, J., Spindler, X., Cantu, A.A., Maynard, P., Lennard, C., and Roux, C., Nile red: Alternative to physical developer for the detection of latent fingermarks on wet porous surfaces? Forensic Science International, 2013. 230(1-3): p. 7480.

4. de la Hunty, M. (2017). An investigation of latent fingermark residues and their development on porous substrates using physical developer and nile red. $\mathrm{PhD}$, University of Technology Sydney, Australia.

5. Houlgrave, S., Andress, M., and Ramotowski, R., Comparison of Different Physical Developer Working Solutions - Part I: Longevity Studies*. Journal of Forensic Identification, 2011. 61(6): p. 621-639.

6. Cantu, A. and Johnson, J., Silver Physical Development of Latent Prints, in Advances in Fingerprint Technology, Second Edition. 2001, CRC Press.

7. Ramotowski, R., Metal Deposition Methods, in Lee and Gaensslen's Advances in Fingerprint Technology, Third Edition. 2012, CRC Press. p. 55-82.

8. de la Hunty, M., Moret, S., Chadwick, S., Lennard, C., Spindler, X., and Roux, C., Understanding Physical Developer (PD): Part II - Is PD targeting eccrine constituents? Forensic Sci Int, 2015.

9. de la Hunty, M., Moret, S., Chadwick, S., Lennard, C., Spindler, X., and Roux, C., Understanding physical developer (PD): Part I - Is PD targeting lipids? Forensic Sci Int, 2015.

10. Moret, S., Personal Communication. 2017

11. Latent Fingerprint Processing Techniques - Selection and Sequencing Guide. [cited 13-12-17]; Chesapeake Bay Division.Available from: http://www.cbdiai.org/Reagents/phydev.html.

12. Ramotowski, R., Personal Communication. 2015

13. Sears, V.G., Personal Communication. 2016 